\title{
Melatonin alleviates hydrogen peroxide induced oxidative damage in MC3T3-E1 cells via SIRT1/p66SHC pathway and promotes osteogenesis
}

Hedong Liu ( $\sim$ liuhedongmed@163.com )

The First Affiliated Hospital of Wannan Medical College: Yijishan Hospital of Wannan Medical College https://orcid.org/0000-0001-8318-1609

\section{Maoxian Ren}

The First Affiliated Hospital of Wannan Medical College: Yijishan Hospital of Wannan Medical College Yang Li

The First Affiliated Hospital of Wannan Medical College: Yijishan Hospital of Wannan Medical College

\section{Ruotian Zhang}

The First Affiliated Hospital of Wannan Medical College: Yijishan Hospital of Wannan Medical College

\section{Nengfeng Ma}

The First Affiliated Hospital of Wannan Medical College: Yijishan Hospital of Wannan Medical College

\section{Tianlin Li}

The First Affiliated Hospital of Wannan Medical College: Yijishan Hospital of Wannan Medical College

\section{Wenkai Jiang}

The First Affiliated Hospital of Wannan Medical College: Yijishan Hospital of Wannan Medical College

\section{Zhi Zhou}

The First Affiliated Hospital of Wannan Medical College: Yijishan Hospital of Wannan Medical College

\section{Xuewei Yao}

The First Affiliated Hospital of Wannan Medical College: Yijishan Hospital of Wannan Medical College

Zhiyi Liu

The First Affiliated Hospital of Wannan Medical College: Yijishan Hospital of Wannan Medical College

Min Yang

The First Affiliated Hospital of Wannan Medical College: Yijishan Hospital of Wannan Medical College

\section{Research Article}

Keywords: melatonin, osteogenesis, osteoporosis, osteoporotic bone defect, ROS, SIRT1/p66SHC pathway

Posted Date: October 15th, 2021 
DOl: https://doi.org/10.21203/rs.3.rs-965478/v1

License: (c) (1) This work is licensed under a Creative Commons Attribution 4.0 International License. Read Full License 


\section{Abstract}

Oxidative stress is an important contributor to the development of osteoporosis. Melatonin, an indoleamine secreted by the pineal gland, has antioxidant properties. This study aims to explore whether melatonin can promote bone formation and elucidate the mechanisms underlying this process. In this study, we used an in vitro hydrogen peroxide $\left(\mathrm{H}_{2} \mathrm{O}_{2}\right)$-induced oxidative stress model in MC3T3-E1 cells and an in vivo ovariectomized osteoporotic bone defect model in rats to explore the protective effects of melatonin against osteoporotic bone defects along with the mechanism underlying these effects. We found that melatonin significantly increased alkaline phosphatase activity, mineralization capacity, and the expression of BMP2, RUNX2, and OPN in MC3T3-E1 cells. Furthermore, melatonin was found to activate SIRT1 and inhibit p66SHC, reduce the intracellular reactive oxygen species levels, stabilize mitochondria, reduce malondialdehyde levels, increase superoxide dismutase activity, and reduce apoptosis in MC3T3-E1 cells treated with $\mathrm{H}_{2} \mathrm{O}_{2}$. Intriguingly, these effects could be reversed by the SIRT1 inhibitor EX527. In vivo experiments confirmed that melatonin improves the microstructure and bone mineral density of the distal femoral bone trabecula and promotes bone formation. Taken together, our findings showed that melatonin can restrain oxidative damage in MC3T3-E1 cells via SIRT1/p66SHC pathway and promote osteogenesis, suggesting that melatonin could be a potential therapeutic agent for osteoporosis-related bone metabolic diseases.

\section{Introduction}

With the increase in life expectancy, osteoporosis has become one of the main health problems worldwide, and its incidence is continuously increasing[1]. Severe bone defects would occur in patients with osteoporosis who were combined with high-energy trauma or infection requiring extensive debridement and bone tumors requiring surgical resection. Moreover, as the incidence of osteoporosis increase, so does the likelihood of osteoporotic bone defects. Healthy bones have a strong regeneration capacity, which allows efficient fracture repair. When large bone defects (above-critical-size bone defect) or complications (associated with osteoporosis and diabetes) occur or individuals have a poor lifestyle (smoking and alcohol abuse), the capacity of bone regeneration reduces. This results in impaired fracture healing, which leads to non-union fractures or bone defects[2]. Although traditional treatments for osteoporosis were partly effective in inhibiting bone loss or promoting bone formation[3], there were many adverse effects, including atypical femur fractures[4], osteonecrosis of the jaw, nausea and dizziness. Therefore, a biologically active substance that can promote bone formation without adverse effects is urgently needed.

Oxidative stress is high in patients with osteoporosis $[5,6]$. It is believed that reactive oxygen species (ROS) occupied an important position in cell damage. Mitochondria, which are the largest and most active producers of ROS, control cell survival and death[7, 8]. When the ROS degradation capacity of the enzymatic hydrolysis system becomes weaker than the cell's ROS generation capability, oxidative stress occurs[9]. Excessive ROS production can lead to the reduced function of osteoblasts and functional activation of osteoclasts, resulting in unbalanced bone remodeling[10]. In addition, mitochondrial 
function is also vulnerable to ROS, such that MC3T3-E1 cells show reduced mitochondrial membrane potential (MMP) and undergo apoptosis after being cultured with antimycin A, a respiratory chain inhibitor that increases ROS production[11].

The production of melatonin, a non-toxic indoleamine secreted by the pineal gland at night, is regulated by the hypothalamus and inhibited by light $[12,13]$. The main function of melatonin is to regulate the circadian rhythm, but it also has anti-aging, anti-oxidation, anti-inflammatory, anti-apoptotic, and reproductive functions[14]. Studies have shown that mitochondria contain the rate-limiting enzyme for the synthesis of melatonin and show high levels of this molecule[15]. Meanwhile, the endosymbiotic theory of the origin of mitochondria postulates that mitochondria originated from melatonin-producing bacteria engulfed by prokaryotes, indicating that mitochondria have the ability to synthesize melatonin[16]. There is evidence that melatonin can activate the expression of SIRT1 in non-tumor cells[17] and that SIRT1 can inhibit p66SHC expression[18, 19]. p66SHC, a member of the mammalian proto-oncogene ShcA protein family, can further activate NADPH oxidase, reduce antioxidant enzyme activity, promote mitochondrial ROS production, and cause oxidative damage[20]. Therefore, we speculated that melatonin could regulate ROS levels by activating the SIRT1/p66SHC pathway, thereby promoting bone formation in osteoblasts and sought to test this hypothesis.

In this study, we used an in vitro model in which oxidative stress was induced in MC3T3-E1 cells using hydrogen peroxide $\left(\mathrm{H}_{2} \mathrm{O}_{2}\right)$ and an in vivo rat ovariectomized osteoporotic bone defect model to explore the protective effects of melatonin against osteoporotic bone defects and the mechanism underlying these effects.

\section{Materials And Methods}

\subsection{Cell culture and differentiation}

MC3T3-E1 cells were cultured in a-MEM with $10 \%$ fetal bovine serum (FBS, Gibco, USA) and 1\% penicillin-streptomycin (Beyotime, China) at $37^{\circ} \mathrm{C}$ in atmosphere containing $5 \% \mathrm{CO}_{2}$. The cells were passaged every 2 days once they grew to approximately $80 \%$ confluence. Osteogenic differentiation was induced with a medium containing $10 \% \mathrm{FBS}, 1 \%$ penicillin-streptomycin, $10 \mathrm{mM} \beta$-glycerophosphate (Solarbio, USA), $50 \mu \mathrm{M}$ ascorbic acid (Solarbio, USA), and $100 \mathrm{nM}$ dexamethasone (Solarbio, USA). In order to examine the effect of melatonin (MLT, Beyotime, China) on osteoblasts treated with $\mathrm{H}_{2} \mathrm{O}_{2}$, cells were incubated in a-MEM alone (control), $\mathrm{H}_{2} \mathrm{O}_{2}$ (pretreated with $400 \mu \mathrm{M} \mathrm{H}_{2} \mathrm{O}_{2}$ for $4 \mathrm{~h}$ and then incubated with a-MEM for $24 \mathrm{~h}$ ), $\mathrm{H}_{2} \mathrm{O}_{2}$ with MLT (pretreated with $400 \mu \mathrm{M} \mathrm{H}_{2} \mathrm{O}_{2}$ for $4 \mathrm{~h}$ then incubated with $100 \mu \mathrm{M}$ melatonin for $24 \mathrm{~h}$ ), $\mathrm{H}_{2} \mathrm{O}_{2}$ with EX527 and MLT (EX527 is a SIRT1 inhibitor, cells were pretreated with 400 $\mu \mathrm{M} \mathrm{H} \mathrm{H}_{2}$ for $4 \mathrm{~h}$, then incubated with $10 \mu \mathrm{M}$ EX527 for $2 \mathrm{~h}$ and $100 \mu \mathrm{M}$ melatonin for $24 \mathrm{~h}$ ).

\subsection{Alkaline phosphatase (ALP) staining and activity assay}


To evaluate the impact of melatonin on the osteogenic differentiation of MC3T3-E1 cells under the influence of $\mathrm{H}_{2} \mathrm{O}_{2}$, ALP staining and activity assays were conducted. Seven days after osteogenic induction, 4\% paraformaldehyde (Beyotime, China) was added to fix the cells for 20 min and then stained with an BCIP/NBT working solution (Beyotime, China). The stained cells were then photographed using a microscope. To assess ALP activity, the Alkaline Phosphatase Assay Kit (Nanjing Jiancheng Bioengineering Institute, China) and BCA Protein Assay Kit (Beyotime, China) were used in accordance with the manufacturer's instructions.

\subsection{Alizarin Red S (ARS) staining and mineralization assay}

Twenty-one days after osteogenic induction, 4\% paraformaldehyde was added to fix the cells for 20 min and then stained with $0.2 \%$ ARS (Solarbio, China). To quantify mineralization, the stained cells were incubated with $10 \%$ cetylpyridinium chloride (Solarbio, USA) for $1 \mathrm{~h}$ and the absorbance was measured by a microplate reader (Bio Tek Instruments, Inc; USA) at $570 \mathrm{~nm}$.

\subsection{Evaluation of intracellular ROS levels}

The cells were washed with PBS and then stained with 2',7'-dichlorodihydro-fluorescein-diacetate (DCFHDA) to detect ROS levels using the Reactive Oxygen Species Assay Kit (KeyGEN BioTECH, China). Images were recorded using a fluorescence microscope (Nikon eclipse Ti-U; USA). Meanwhile, the mean fluorescence intensity was measured using flow cytometry (FC500MPL, Beckman Coulter, USA) to evaluate the levels of ROS.

\subsection{Assays for malondialdehyde (MDA) levels and superoxide dismutase (SOD) activity}

MDA and SOD, both biomarkers of oxidative stress, were detected using the Cell Malondialdehyde assay kit and Superoxide Dismutase assay kit (Nanjing Jiancheng Bioengineering Institute, China), respectively, in accordance with the manufacturer's instructions. The protein concentration of each sample was measured using a BCA Protein Assay Kit. MDA concentrations were manifested as nmol/mg protein and SOD activity as $\mathrm{U} / \mathrm{mg}$ protein.

\subsection{Detection of mitochondrial membrane potential (MMP)}

JC-1 is a sensitive indicator of MMP. When MMP is high, the JC-1 fluorescent probe emits red fluorescence, but when it is low, this probe emits green fluorescence. Low MMP implies cell apoptosis. JC1 aggregates and $\mathrm{JC}-1$ monomers were detected in treated cells and photographed using a fluorescence microscope. The acquired images were further assessed using Image $\mathrm{J}$ software (version $1.52 \mathrm{v}, \mathrm{NIH}$, USA).

\subsection{Analysis of apoptosis}

The Annexin V-FITC/PI Apoptosis Detection Kit (KeyGEN BioTECH, China) was used to detect the effect of different treatments on apoptosis. Stain cells with Annexin V and PI and then subjected to flow cytometry. Positive Annexin V staining and negative PI staining indicated viable apoptotic cells, whereas double 
positive staining indicated non-viable apoptotic cells. The sum of the two counts was used to calculate the apoptosis rate.

\subsection{Western blotting}

Treated cells were lysed on ice using RIPA buffer (Beyotime, China) containing a protease inhibitor (Beyotime, China), and the protein concentration was measured using the BCA Protein Assay Kit. The proteins were separated by SDS-PAGE and transferred to PVDF membranes. The membranes were blocked with bovine serum albumin for $2 \mathrm{~h}$ and then incubated with the following primary antibodies overnight at $4{ }^{\circ} \mathrm{C}$ : BMP2 (1:1000, AF5163, Affinity, USA), RUNX2 (1:1000, AF5186, Affinity, USA), OPN (1:1000, AF0227, Affinity, USA), Bcl2 (1:1000, AF6139, Affinity, USA), Bax (1:1000, AF0120, Affinity, USA), cleaved caspase3 (1:1000, AF7022, Affinity, USA), caspase3 (1:1000,AF6311,Affinity,USA), SIRT1 (1:1000, DF6033, Affinity, USA), p66SHC (1:1000,AF6245,Affinity,USA) and $\beta$-actin (1:10000, AF7018, Affinity, USA). The following day, the membranes were incubated with HRP-labeled secondary antibodies (1:5000, S0001, Affinity, USA) for $2 \mathrm{~h}$. Then, the bands were developed using an enhanced chemiluminescence kit (KF005, Affinity, USA) and quantified by Image $J$ software.

\subsection{Experimental animals}

All animal experiments were approved by the Ethical Committee of Yijishan Hospital. Forty eight-week-old female Sprague Dawley rats weighing 220-240 g were housed in the Central laboratory of Yijishan Hospital for groups of four. The temperature was controlled at $22 \pm 2^{\circ} \mathrm{C}$, the humidity was controlled at $50 \%$, the light cycle was controlled at $12 \mathrm{~h}$ light/dark and all the rats had free access to food and water.

\subsection{Surgery and treatment}

The standard method was used for bilateral ovariectomy $(n=25)$ and sham surgery $(n=15)$ [21]. After being ovariectomized (OVX), rats were fed normally for three months until the osteoporosis model was established. Then, five rats each from the OVX and sham group were sacrificed. Micro-computed tomography (micro-CT, SCANCO $\mu \mathrm{CT}-100$, Switzerland) was used to measure the bone mineral density (BMD) of the left femur in each rat, and the right femur was used for hematoxylin-eosin (HE) staining to verify the successful establishment of the osteoporosis model. Once the osteoporosis model was established, all animals underwent surgery to induce bilateral femur defects according to the standard protocol[21]. After the operation, the rats were segregated into three groups: control group ( $n=10$, sham, bone defect surgery, and intraperitoneal injection of the same amount of saline from 4 to 6 pm daily), OVX group ( $n=10$, ovariectomy, bone defect surgery, and intraperitoneal injection of the same amount of saline from 4 to 6 pm daily), and melatonin (MLT) group ( $n=10$, ovariectomy, bone defect surgery, and intraperitoneal injection of $50 \mathrm{mg} / \mathrm{kg}$ melatonin from 4 to $6 \mathrm{pm}$ daily). All rats were sacrificed two months later, the left femur was examined using micro-CT, and the right femur was subjected to HE staining, Masson's trichrome staining, and immunohistochemical staining after decalcification.

\subsection{Micro-CT analysis}


To analyze the microstructure of the femoral metaphysis, micro-CT was used and the microarchitecture of the defect zone was evaluated. The following parameters were used: scanning voltage, $70 \mathrm{kV}$; current, $200 \mu \mathrm{A}$; exposure time, $300 \mathrm{~ms}$; and thickness, $15 \mu \mathrm{m}$. To assess bone regeneration, a 2.0-mm-diameter circular area containing the bone defect zone was selected as the volume of interest. Meanwhile, BMD, bone volume fraction (BV/TV), trabecular number (Tb.N), trabecular thickness (Tb.Th) and trabecular separation (Tb.Sp) were measured using built-in software after 3D reconstruction.

\subsection{Histological and immunohistochemical Analysis}

Histological and immunohistochemical analysis were conducted after two months of drug treatment. After fixation, decalcification, and paraffin embedding, the femur was incised into $5 \mu \mathrm{m}$ slices and stained with HE and Masson's trichrome stains for morphologic assessment. New bone formation after bone injuries was examined using a light microscope (Nikon eclipse Ti-U; USA). After Masson's trichrome staining, new bone tissue and collagen fibers were stained blue, and mature bone and muscle fibers were stained red. The new bone formation in defect area was measured by Image $\mathrm{J}$ software (version $1.52 \mathrm{v}$, $\mathrm{NIH}, \mathrm{USA})$.

The expression levels of osteocalcin (OCN) and type 1 collagen (COL1) in the femur were detected using immunohistochemistry. Briefly, slices were deparaffinized with xylene, immersed in $3 \% \mathrm{H}_{2} \mathrm{O}_{2}$ to remove endogenous catalase, blocked against non-specific antibodies, and then incubated with specific primary antibodies (OCN, 1:200, DF12303, Affinity; COL1, 1:100, AF7001, Affinity) overnight at $4^{\circ} \mathrm{C}$. The next day, the slices were incubated with the secondary antibody and subjected to DAB staining, then counterstained with hematoxylin, dehydrated, and mounted. Antigen-expressing positive cells were stained brown. Photographs were obtained under a light microscope. The average optical density of OCN and COL1 were measured by Image $\mathrm{J}$ software.

\subsection{Statistical analysis}

All values were expressed as mean \pm standard deviation (SD), and all statistical analysis were performed using GraphPad Prism 8 (USA). One-way analysis of variance (ANOVA) was used for comparisons among multiple groups, and independent samples t-tests were used for comparisons between two groups. $\mathrm{P} \otimes 0.05$ was considered statistically significant.

\section{Results}

\subsection{Melatonin antagonizes $\mathrm{H}_{2} \mathrm{O}_{2}$-induced effects in MC3T3- E1 cells}

We investigated which $\mathrm{H}_{2} \mathrm{O}_{2}$-induced effects were affected by melatonin in MC3T3-E1 cells by examining cell differentiation and mineralization capacity. We also detected the expression of BMP2, RUNX2, and OPN, all of which are involved in bone formation. The results showed that the ALP-positive areas 
(Fig. 1a), ALP activity (Fig. 1b), calcium deposition (Fig. 1c) and quantitative analysis (Fig. 1d), and levels of osteogenic proteins (Fig. $1 \mathrm{f}, \mathrm{g}$ and $\mathrm{h}$ ) were lower in the $\mathrm{H}_{2} \mathrm{O}_{2}$ group than in the control group. In addition, we found that $\mathrm{H}_{2} \mathrm{O}_{2}$ pretreatment reduced SIRT1 expression (Fig. 1i) while increased p66SHC expression (Fig. 1j). However, in MC3T3-E1 cells treated with melatonin, these values had all changed in the opposite direction. Melatonin reversed the reduction of SIRT1 and the increase of p66SHC induced by $\mathrm{H}_{2} \mathrm{O}_{2}$ treatment. Interestingly, incubated with the SIRT1 inhibitor EX527 decreased ALP expression, ALP activity, calcium deposition, and the levels of osteogenic proteins. Particularly, EX527 inhibited the effect of melatonin on p66SHC and thus increased the expression of p66SHC. Together, these data suggest that melatonin improved the function of $\mathrm{H}_{2} \mathrm{O}_{2}$-treated MC3T3-E1 cells via SIRT1/p66SHC pathway.

\subsection{Melatonin inhibits the $\mathrm{H}_{2} \mathrm{O}_{2}$-induced apoptosis of MC3T3-E1 cells and increases MMP}

The apoptosis of osteoblasts is a significant contributor to postmenopausal osteoporosis. Therefore, we examined whether melatonin affects the apoptosis of MC3T3-E1 cells and also examined the levels of apoptosis-associated proteins. As shown in Fig. 2, $\mathrm{H}_{2} \mathrm{O}_{2}$ treatment significantly increased the rate of apoptosis (Fig. 2a and b). Western blotting results also confirmed that $\mathrm{H}_{2} \mathrm{O}_{2}$ increased cell apoptosis (Fig. 2C). Specifically, $\mathrm{H}_{2} \mathrm{O}_{2}$ pretreatment increased the expression of cleaved caspase3 (Fig. 2d) and Bax (Fig. 2e) and decreased the expression of $\mathrm{Bcl} 2$ (Fig. 2f). We further investigated the MMP and found that $\mathrm{H}_{2} \mathrm{O}_{2}$ reduced the MMP, causing the $\mathrm{JC}-1$ fluorescent probe to emit green light, and the red/green fluorescent ratio was reduced (Fig. 3a and b), which indicated that the cells underwent apoptosis. Whereas managed with melatonin reversed these changes and the SIRT1 inhibitor EX527 attenuated the antiapoptotic effects of melatonin. These data imply that melatonin alleviated the $\mathrm{H}_{2} \mathrm{O}_{2}$-induced apoptosis of MC3T3-E1 cells via the SIRT1/p66SHC pathway.

\subsection{Melatonin enhances the antioxidant capacity of $\mathrm{H}_{2} \mathrm{O}_{2}-$ treated MC3T3-E1 cells}

Next, we strove to clarify whether the protective function of melatonin in MC3T3-E1 cells was linked to its antioxidant properties by examining the levels of ROS, SOD, and MDA (a product of lipid peroxidation). We found that $\mathrm{H}_{2} \mathrm{O}_{2}$ increased the level of ROS and this result was detected by both fluorescence microscopy (Fig. 4a) and flow cytometry (Fig. 4b and c). Meanwhile, $\mathrm{H}_{2} \mathrm{O}_{2}$ increased MDA (Fig. 4d) level in cells and reduced the activity of SOD (Fig. 4e), indicating that oxidative stress increased in MC3T3-E1 cells after pretreated with $\mathrm{H}_{2} \mathrm{O}_{2}$. Conversely, when cells were handled with melatonin, ROS and MDA levels decreased and SOD activity increased. The SIRT1 inhibitor EX527 reversed the antioxidant effects of melatonin. Hence, melatonin appeared to regulate the antioxidant capacity of MC3T3-E1 cells through the SIRT1/p66SHC pathway.

\subsection{Validation of the rat osteoporosis model}


Histological images and micro-CT data were shown in Figure 5. micro-CT showed that the Tb.N and Tb.Th in the OVX group were lower than those in the sham group, while Tb.Sp showed the opposite trend (Fig. 5a and b). HE staining confirmed that the BMD was lower in the OVX group than in the sham group (Fig. 5c). Together, the findings confirmed the successful establishment of the rat osteoporosis model.

\subsection{Melatonin improves bone mass and bone microstructure in OVX rats}

We assessed the impact of melatonin on trabecular bone mass and microstructure in rats with osteoporotic bone defects. The results of 3D micro-CT reconstruction were shown in Fig. 6 . The findings indicated that after 20 weeks, bone formation in the bone defect zone was significantly greater in the MLT group than in the OVX group, although it was less than that in the control group (Fig. 6a). Bone formation in the bone defect zone was the highest in the control group, while it was almost negligible in the OVX group. Specifically, the BMD (Fig. 6b), BV/TV (Fig. 6c), Tb.N (Fig. 6d), and Tb.Th (Fig. 6e) in the MLT group were higher than those in the OVX group and lower than those in the control group, with Tb.Sp (Fig. 6f) showing the opposite trend. These results attested that melatonin can partially promote the healing of osteoporotic bone defects and the formation of new bone.

To further examine the morphology of bone healing in the defect zone, histological analysis was performed. HE staining showed that the Tb.N in the bone defect zone was higher in the MLT group than in the OVX group but lower than that in the control group (Fig. 7a). Masson's trichrome staining showed that the bone defect zone in the MLT group was dominated by new bone tissue and collagen fibers, while that in the control group was populated by mature bone, new bone, and collagen fibers. However, almost no new bone tissue was observed in the OVX group (Fig. 7b). These results proved that the control group showed the highest rate of new bone formation, followed by the MLT group and the OVX group (Fig. 7e), consistent with our micro-CT findings.

The expression of OCN and COL1 in the femur was detected using immunohistochemistry. As shown in Fig. 7, the expression levels of OCN and COL 1 in trabecular bone were highest in the MLT group, while the OVX group had almost no expression (Fig. 7c and d). Quantitative analysis showed that the expression levels of OCN and COL1 in the MLT group were higher than those in the control group (Fig. 7f and g). We speculated that this was related to the high new bone formation rate in the control group, suggesting that in this group, most of the bone had already matured.

\section{Discussion}

There are growing evidences that oxidative stress plays an important role in the pathogenesis of osteoporosis[22, 23]. Our study also confirmed that $\mathrm{H}_{2} \mathrm{O}_{2}$ can weaken the ALP activity and mineralization ability of MC3T3-E1 cells. Although studies have shown that melatonin can exert antioxidant effects by regulating SIRT1, the specific mechanism remains to be studied[24]. In our study, we found that melatonin can attenuate the oxidative stress damage of MC3T3-E1 cells induced by $\mathrm{H}_{2} \mathrm{O}_{2}$, which involves 
the down-regulation of the adaptor protein $\mathrm{p} 66 \mathrm{SHC}$ and the enhancement of mitochondrial function. Particularly, based on the evidence of the SIRT1 inhibitor EX527, we found that melatonin depressed the oxidative stress of MC3T3-E1 cells by regulating the SIRT1/p66SHC pathway and promotes osteogenesis.

As an isoform of ShcA gene family, p66SHC occupied an important position in oxidative stress and apoptosis[25,26]. When cells experience oxidative stress, p66SHC transfer to mitochondria and oxidize cytochrome $\mathrm{C}$, reducing oxygen to $\mathrm{H}_{2} \mathrm{O}_{2}$, leading to increased ROS which can promote the opening of mitochondrial permeability conversion pore, reduce the activity of electron transfer chain protein complex and release pro-apoptotic factors to cause apoptosis[27, 28]. Studies have shown that superoxide production was remarkably reduced in p66SHC knockout mice[29]. Maria et al. also confirmed that with the increase of months, C57BL/ 6 mice showed a decrease in bone strength and bone density, accompanied by an increase in the level of oxidative stress, and an increase in the phosphorylation levels of p53 and p66SHC[30]. In our research, we found that the expression level of p66SHC protein increased in the model of $\mathrm{H}_{2} \mathrm{O}_{2}$-induced oxidative stress damage in MC3T3-E1 cells, revealing that $\mathrm{p} 66 \mathrm{SHC}$ was a possible target for the intervention of oxidative stress in MC3T3-E1 cells. In addition, previous studies have shown that in mammals, with the increase of age, the content of melatonin gradually decreases[31]. Based on the evidences that melatonin was produced in mitochondria and taken part in anti-oxidative stress $[15,16]$, in the meantime, p66SHC needs to be transferred to mitochondria to induce oxidative stress, we considered that the anti-oxidative stress effect of melatonin was associated with p66SHC. In our study, we found that the increased expression of p66SHC protein was accompanied by the decreased expression of SIRT1 in MC3T3-E1 cells pretreated with $\mathrm{H}_{2} \mathrm{O}_{2}$, and this effect could be alleviated by melatonin intervention. Further studies showed that SIRT1 inhibitor EX527 reversed the protective effect of melatonin on $\mathrm{H}_{2} \mathrm{O}_{2}$-induced MC3T3-E1 cells oxidative stress damage and increased the expression of p66SHC. In fact, SIRT1, as an NAD+-dependent type III histone deacetylase, was widely involved in the regulation of physiological processes such as cell cycle, inflammation, apoptosis, and autophagy[32]. In endothelial cells, SIRT1 overexpression can reduce the binding of acetylated histone $\mathrm{H} 3$ to the p66SHC promoter, thereby decrease the transcription of p66SHC[33]. The present study demonstrated that melatonin protected $\mathrm{H}_{2} \mathrm{O}_{2}$-induced oxidative stress damage in MC3T3-E1 cells via SIRT1/p66SHC pathway.

Melatonin is a non-toxic indolamine secreted by the pineal gland and regulated by the hypothalamus, which mainly regulates circadian rhythm[34]. There are increasing evidences that melatonin - as an antioxidant - can not only anti-inflammatory, antioxidant, anti-apoptosis and regulate reproductive function, but also restrain tumor cell proliferation[35] and regulate the expression of cell adhesion molecules related to tight junctions and adhesion junctions between tumor cells to restrain tumor metastasis[36, 37]. In this study, we determined the in vitro cytological study of $\mathrm{H}_{2} \mathrm{O}_{2}$-induced oxidative stress damage in MC3T3-E1 cells by measuring oxidative stress and apoptosis-related indicators. Melatonin management remarkably reduced $\mathrm{H}_{2} \mathrm{O}_{2}$-induced oxidative stress damage of MC3T3-E1 cells by increasing the activity of SOD and decreasing the content of MDA. Meanwhile, melatonin protected 
MC3T3-E1 cells from oxidative stress damage by removing $\mathrm{H}_{2} \mathrm{O}_{2}$-induced ROS production. Evidences indicated that $\mathrm{H}_{2} \mathrm{O}_{2}$-induced ROS production was associated with the decrease of MMP, which can promote mitochondrial cytochrome $\mathrm{C}$ release, activate caspase-3, and cause osteoblast apoptosis[38]. Furthermore, mitochondrial remodeling has also shown circadian rhythm and can be tightly regulated by melatonin-governed proteins[39]. In this study, we found that $\mathrm{H}_{2} \mathrm{O}_{2}$ can significantly reduce MMP, leading to MC3T3-E1 cells apoptosis, resulting in decreased ALP activity, insufficient mineralization ability, and decreased expression of osteoblast-related proteins, while melatonin management can significantly increase MMP and reduce MC3T3-E1 cells apoptosis. Those data implied that melatonin restrained $\mathrm{H}_{2} \mathrm{O}_{2}$ induced mitochondrial dysfunction in a SIRT1-dependent manner, which has also been verified in other cell types[40, 41]. Given that p66SHC was governed by SIRT1 and melatonin can up-regulate SIRT1, we speculated that the protective effect of melatonin on $\mathrm{H}_{2} \mathrm{O}_{2}$-induced oxidative stress damage was related to SIRT1's inhibition of p66SHC expression and the enhancement of mitochondrial function, it was also confirmed by SIRT1 inhibitor EX527 which can reverse the function of melatonin.

Ovariectomized rats experience significant bone loss similar to that observed in postmenopausal osteoporosis. To verify the effect of melatonin on bone formation, we established an osteoporotic bone defect model. The BV/TV value was considered to be the most effective analysis tool for micro-CT to measure bone defects because it can directly reflect the amount of bone formation[42]. Meanwhile, HE staining can visually observe the number, thickness and spacing of bone trabeculae, and Masson staining can distinguish new bone from mature bone. Therefore, we used micro-CT, HE staining and Masson staining to observe the bone regeneration in the bone defect area. The results showed that melatonin could promote the bone healing of osteoporotic bone defects, but its effect was not as good as that of the control group. Immunohistochemical results also showed that the expression of OCN and COL1 in MLT group was higher than that in OVX group. Those data indicating that the role of estrogen in the treatment of osteoporosis cannot be replaced by melatonin, but melatonin can be used as an adjunctive drug for the treatment of osteoporotic bone metabolic diseases.

Our research has some limitations. First, although inflammation can promote osteoporosis to a certain degree[43], we only studied the antioxidant and anti-apoptotic properties of melatonin and did not examine whether its anti-inflammatory effects can reduce osteoporosis. Second, even though osteoporosis is caused by the imbalance between osteoblast bone formation and osteoclast bone resorption, we only studied the effect of melatonin on osteoblasts and its effect on osteoclasts was not investigated. In our subsequent research, we will focus on these two issues.

In conclusion, the present study suggests that melatonin can alleviates $\mathrm{H}_{2} \mathrm{O}_{2}$-induced oxidative damage in MC3T3-E1 cells via SIRT1/p66SHC pathway and promotes osteogenesis. SIRT1 activation inhibits the expression of p66SHC, reduces the production of ROS and MDA, increases the activity of SOD, helps maintain the integrity of mitochondria, alleviates oxidative stress, and inhibits the apoptosis caused by oxidative damage. Our research provides strong evidence supporting the potential of melatonin as a treatment agent for osteoporosis-related bone metabolism diseases. 


\section{Declarations}

\section{Author contribution}

All authors contributed to the study conception and design. Material preparation, data collection and analysis were performed by Hedong Liu, and Maoxian Ren. The first draft of the manuscript was written by Hedong Liu and Maoxian Ren and all authors commented on previous versions of the manuscript. All authors read and approved the final manuscript.

\section{Fundings}

This research was supported by the National Natural Science Foundation of China (81341054, 81171732), Talented Scholars of Wannan Medical College (YR201917), Technology Mountaineering Program of Yijishan Hospital, and Wannan Medical College (PF2019005).

\section{Data Availability}

The data supporting the results of this study can be obtained from the corresponding author upon request.

\section{Conflict of interests}

The authors declare they have no conflict of interests.

\section{References}

1. Lin X, Xiong D, Peng YQ, Sheng ZF, Wu XY, Wu XP, Wu F, Yuan LQ, Liao EY (2015) Epidemiology and management of osteoporosis in the People's Republic of China: current perspectives. Clin Interv Aging 10:1017-1033. .https://doi.org/10.2147/CIA.S54613

2. Ho-Shui-Ling A, Bolander J, Rustom LE, Johnson AW, Luyten FP, Picart C (2018) Bone regeneration strategies: Engineered scaffolds, bioactive molecules and stem cells current stage and future perspectives. Biomaterials 180:143-162. https://doi.org/10.1016/j.biomaterials.2018.07.017

3. Kanis JA, Cooper C, Rizzoli R, Reginster JY, Scientific Advisory Board of the European Society for C, Economic Aspects of $\mathrm{O}$, the Committees of Scientific A and National Societies of the International Osteoporosis F (2019) European guidance for the diagnosis and management of osteoporosis in postmenopausal women. Osteoporos Int 30:3-44.https://doi.org/10.1007/s00198-018-4704-5

4. Starr J, Tay YKD, Shane E (2018) Current Understanding of Epidemiology, Pathophysiology, and Management of Atypical Femur Fractures. Curr Osteoporos Rep 16:519-529. https://doi.org/10.1007/s11914-018-0464-6

5. Shen CL, Yang S, Tomison MD, Romero AW, Felton CK, Mo H (2018) Tocotrienol supplementation suppressed bone resorption and oxidative stress in postmenopausal osteopenic women: a 12-week 
randomized double-blinded placebo-controlled trial. Osteoporos Int 29:881-891. .https://doi.org/10.1007/s00198-017-4356-x

6. Yang S, Feskanich D, Willett WC, Eliassen AH, Wu T (2014) Association between global biomarkers of oxidative stress and hip fracture in postmenopausal women: a prospective study. Journal of bone mineral research: the official journal of the American Society for Bone Mineral Research 29:25772583. https://doi.org/10.1002/jbmr.2302

7. Venditti P, Di Meo S (2020) The Role of Reactive Oxygen Species in the Life Cycle of the Mitochondrion. Int J Mol Sci 21. https://doi.org/10.3390/ijms21062173

8. Hadrava Vanova K, Kraus M, Neuzil J, Rohlena J (2020) Mitochondrial complex II and reactive oxygen species in disease and therapy. Redox Rep 25:26-32.

.https://doi.org/10.1080/13510002.2020.1752002

9. Liguori I, Russo G, Curcio F, Bulli G, Aran L, Della-Morte D, Gargiulo G, Testa G, Cacciatore F, Bonaduce D, Abete P (2018) Oxidative stress, aging, and diseases. Clin Interv Aging 13:757-772. https://doi.org/10.2147/CIA.S158513

10. Tao H, Ge G, Liang X, Zhang W, Sun H, Li M, Geng D (2020) ROS signaling cascades: dual regulations for osteoclast and osteoblast. Acta Biochim Biophys Sin (Shanghai) 52:1055-1062. https://doi.org/10.1093/abbs/gmaa098

11. Choi EM, Lee YS (2011) Mitochondrial defects and cytotoxicity by antimycin A on cultured osteoblastic MC3T3-E1 cells. Food Chem Toxicol 49:2459-2463. https://doi.org/10.1016/j.fct.2011.04.037

12. Tamura H, Jozaki M, Tanabe M, Shirafuta Y, Mihara Y, Shinagawa M, Tamura I, Maekawa R, Sato S, Taketani T, Takasaki A, Reiter RJ, Sugino N (2020) Importance of Melatonin in Assisted Reproductive Technology and Ovarian Aging. Int J Mol Sci 21. https://doi.org/10.3390/ijms21031135

13. Li T, Jiang S, Lu C, Yang W, Yang Z, Hu W, Xin Z, Yang Y (2019) Melatonin: Another avenue for treating osteoporosis? Journal of pineal research 66:e12548. https://doi.org/10.1111/jpi.12548

14. Hardeland R (2019) Aging, Melatonin, and the Pro- and Anti-Inflammatory Networks. Int J Mol Sci 20. https://doi.org/10.3390/ijms20051223

15. Tan D-X, Manchester LC, Liu X, Rosales-Corral SA, Acuna-Castroviejo D, Reiter RJ (2013) Mitochondria and chloroplasts as the original sites of melatonin synthesis: a hypothesis related to melatonin's primary function and evolution in eukaryotes. Journal of pineal research 54:127-138. https://doi.org/10.1111/jpi.12026

16. Reiter RJ, Rosales-Corral S, Tan DX, Jou MJ, Galano A, Xu B (2017) Melatonin as a mitochondriatargeted antioxidant: one of evolution's best ideas. Cell Mol Life Sci 74:3863-3881. https://doi.org/10.1007/s00018-017-2609-7

17. Zhang Z, Lin J, Tian N, Wu Y, Zhou Y, Wang C, Wang Q, Jin H, Chen T, Nisar M, Zheng G, Xu T, Gao W, Zhang X, Wang X (2019) Melatonin protects vertebral endplate chondrocytes against apoptosis and calcification via the Sirt1-autophagy pathway. J Cell Mol Med 23:177-193.

https://doi.org/10.1111/jcmm.13903

Page 13/21 
18. Wang $D$, Wang $T$, Wang $R$, Zhang $X$, Wang L, Xiang Z, Zhuang L, Shen S, Wang H, Gao Q, Wang $Y$ (2020) Suppression of p66Shc prevents hyperandrogenism-induced ovarian oxidative stress and fibrosis. J Transl Med 18:84. https://doi.org/10.1186/s12967-020-02249-4

19. Qu B, Gong K, Yang H, Li Y, Jiang T, Zeng Z, Cao Z, Pan X (2018) SIRT1 suppresses high glucose and palmitate-induced osteoclast differentiation via deacetylating p66Shc. Mol Cell Endocrinol 474:97104. .https://doi.org/10.1016/j.mce.2018.02.015

20. Kong X, Guan J, Li J, Wei J, Wang R (2017) P66-SIRT1 Regulation of Oxidative Stress Protects Against Cardio-cerebral Vascular Disease. Mol Neurobiol 54:5277-5285. https://doi.org/10.1007/s12035-016-0073-2

21. Xie Z, Weng S, Li H, Yu X, Lu S, Huang K, Wu Z, Bai B, Boodhun V, Yang L (2017) Teriparatide promotes healing of critical size femur defect through accelerating angiogenesis and degradation of $\beta$-TCP in OVX osteoporotic rat model. Biomedicine pharmacotherapy = Biomedecine pharmacotherapie 96:960-967. .https://doi.org/10.1016/j.biopha.2017.11.141

22. Manolagas SC (2010) From estrogen-centric to aging and oxidative stress: a revised perspective of the pathogenesis of osteoporosis. Endocr Rev 31:266-300. https://doi.org/10.1210/er.2009-0024

23. Kimball JS, Johnson JP, Carlson DA (2021) Oxidative Stress and Osteoporosis. J Bone Joint Surg Am 103:1451-1461. https://doi.org/10.2106/JBJS.20.00989

24. Chen W, Chen X, Chen AC, Shi Q, Pan G, Pei M, Yang H, Liu T, He F (2020) Melatonin restores the osteoporosis-impaired osteogenic potential of bone marrow mesenchymal stem cells by preserving SIRT1-mediated intracellular antioxidant properties. Free Radic Biol Med 146:92-106. .https://doi.org/10.1016/j.freeradbiomed.2019.10.412

25. Xiao Y, Xia J, Cheng J, Huang H, Zhou Y, Yang X, Su X, Ke Y, Ling W (2019) Inhibition of SAdenosylhomocysteine Hydrolase Induces Endothelial Dysfunction via Epigenetic Regulation of p66shc-Mediated Oxidative Stress Pathway. Circulation 139:2260-2277. https://doi.org/10.1161/CIRCULATIONAHA.118.036336

26. Natalicchio A, Tortosa F, Perrini S, Laviola L, Giorgino F (2011) p66Shc, a multifaceted protein linking Erk signalling, glucose metabolism, and oxidative stress. Arch Physiol Biochem 117:116-124. .https://doi.org/10.3109/13813455.2011.562513

27. Giorgio M, Migliaccio E, Orsini F, Paolucci D, Moroni M, Contursi C, Pelliccia G, Luzi L, Minucci S, Marcaccio M, Pinton P, Rizzuto R, Bernardi P, Paolucci F, Pelicci PG (2005) Electron transfer between cytochrome $\mathrm{c}$ and p66Shc generates reactive oxygen species that trigger mitochondrial apoptosis. Cell 122:221-233

28. Orsini F, Migliaccio E, Moroni M, Contursi C, Raker VA, Piccini D, Martin-Padura I, Pelliccia G, Trinei M, Bono M, Puri C, Tacchetti C, Ferrini M, Mannucci R, Nicoletti I, Lanfrancone L, Giorgio M, Pelicci PG (2004) The life span determinant p66Shc localizes to mitochondria where it associates with mitochondrial heat shock protein 70 and regulates trans-membrane potential. J Biol Chem 279:25689-25695. https://doi.org/10.1074/jbc.M401844200 
29. Tomilov AA, Bicocca V, Schoenfeld RA, Giorgio M, Migliaccio E, Ramsey JJ, Hagopian K, Pelicci PG, Cortopassi GA (2010) Decreased superoxide production in macrophages of long-lived p66Shc knockout mice. J Biol Chem 285:1153-1165. https://doi.org/10.1074/jbc.M109.017491

30. Almeida M, Han L, Martin-Millan M, Plotkin LI, Stewart SA, Roberson PK, Kousteni S, O'Brien CA, Bellido T, Parfitt AM, Weinstein RS, Jilka RL, Manolagas SC (2007) Skeletal involution by ageassociated oxidative stress and its acceleration by loss of sex steroids. J Biol Chem 282:2728527297. https://doi.org/10.1074/jbc.M702810200

31. Amstrup AK, Sikjaer T, Mosekilde L, Rejnmark L (2013) Melatonin and the skeleton. Osteoporos Int 24:2919-2927. .https://doi.org/10.1007/s00198-013-2404-8

32. Chen C, Zhou M, Ge Y, Wang X (2020) SIRT1 and aging related signaling pathways. Mech Ageing Dev 187:111215. https://doi.org/10.1016/j.mad.2020.111215

33. Zhou S, Chen H-Z, Wan Y-Z, Zhang Q-J, Wei Y-S, Huang S, Liu J-J, Lu Y-B, Zhang Z-Q, Yang R-F, Zhang R, Cai H, Liu D-P, Liang C-C (2011) Repression of P66Shc expression by SIRT1 contributes to the prevention of hyperglycemia-induced endothelial dysfunction. Circulation research 109:639-648. https://doi.org/10.1161/CIRCRESAHA.111.243592

34. Stacchiotti A, Favero G, Rodella LF (2020) Impact of Melatonin on Skeletal Muscle and Exercise. Cells 9.https://doi.org/10.3390/cells9020288

35. Kvietkauskas M, Zitkute V, Leber B, Strupas K, Stiegler P, Schemmer P (2020) The role of melatonin in colorectal cancer treatment: a comprehensive review. Ther Adv Med Oncol 12:1758835920931714. https://doi.org/10.1177/1758835920931714

36. Akbarzadeh M, Movassaghpour AA, Ghanbari H, Kheirandish M, Fathi Maroufi N, Rahbarghazi R, Nouri M, Samadi N (2017) The potential therapeutic effect of melatonin on human ovarian cancer by inhibition of invasion and migration of cancer stem cells. Sci Rep 7:17062. https://doi.org/10.1038/s41598-017-16940-y

37. Cos S, Fernandez R, Guezmes A, Sanchez-Barcelo EJ (1998) Influence of melatonin on invasive and metastatic properties of MCF-7 human breast cancer cells. Cancer Res 58:4383-4390

38. Zhu FB, Wang JY, Zhang YL, Hu YG, Yue ZS, Zeng LR, Zheng WJ, Hou Q, Yan SG, Quan RF (2016) Mechanisms underlying the antiapoptotic and anti-inflammatory effects of monotropein in hydrogen peroxide-treated osteoblasts. Mol Med Rep 14:5377-5384. https://doi.org/10.3892/mmr.2016.5908

39. Neufeld-Cohen A, Robles MS, Aviram R, Manella G, Adamovich Y, Ladeuix B, Nir D, Rousso-Noori L, Kuperman Y, Golik M, Mann M, Asher G (2016) Circadian control of oscillations in mitochondrial ratelimiting enzymes and nutrient utilization by PERIOD proteins. Proc Natl Acad Sci U S A 113:E1673E1682. .https://doi.org/10.1073/pnas.1519650113

40. Guo Y, Sun J, Bu S, Li B, Zhang Q, Wang Q, Lai D (2020) Melatonin protects against chronic stressinduced oxidative meiotic defects in mice MIl oocytes by regulating SIRT1. Cell Cycle 19:1677-1695. https://doi.org/10.1080/15384101.2020.1767403

41. Nopparat C, Sinjanakhom P, Govitrapong P (2017) Melatonin reverses H O -induced senescence in SH-SY $5 Y$ cells by enhancing autophagy via sirtuin 1 deacetylation of the RelA/p65 subunit of NF-KB. 
Journal of pineal research 63. https://doi.org/10.1111/jpi.12407

42. Wan J, Ma T, Jin Y, Qiu S (2020) The effects of morin on bone regeneration to accelerate healing in bone defects in mice. Int J Immunopathol Pharmacol 34:2058738420962909.

https://doi.org/10.1177/2058738420962909

43. Michalski MN, McCauley LK (2017) Macrophages and skeletal health. Pharmacol Ther 174:43-54. .https://doi.org/10.1016/j.pharmthera.2017.02.017

\section{Figures}
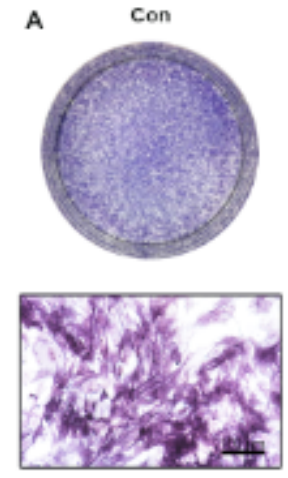

C Con
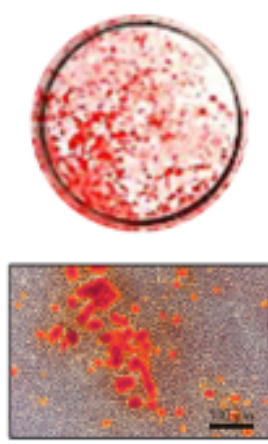

E
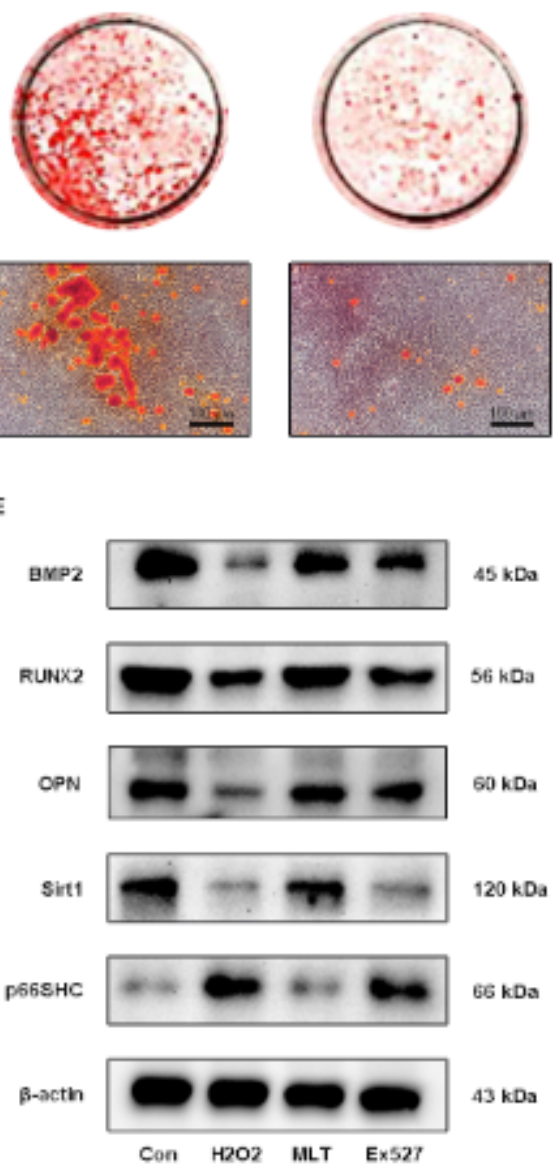

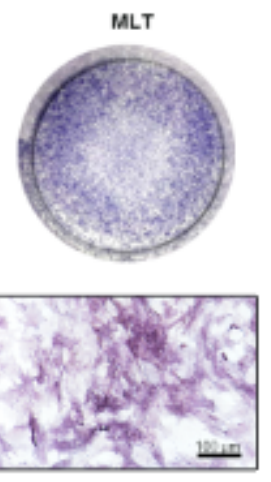

MLT
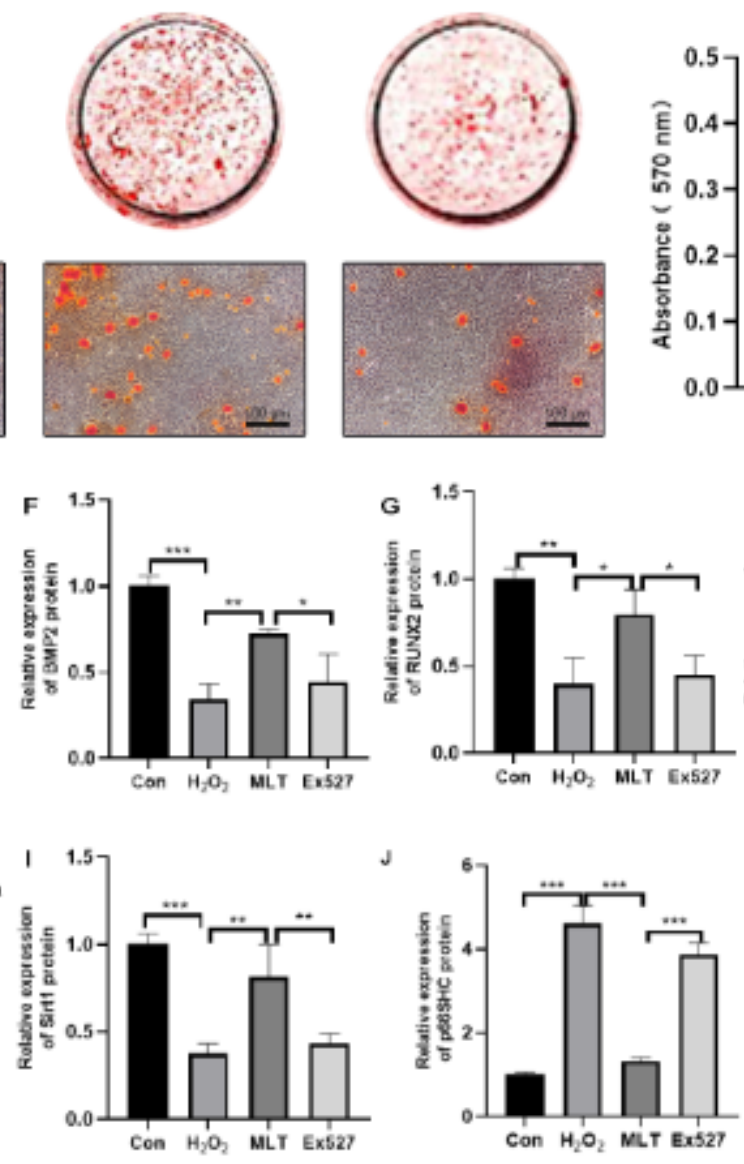
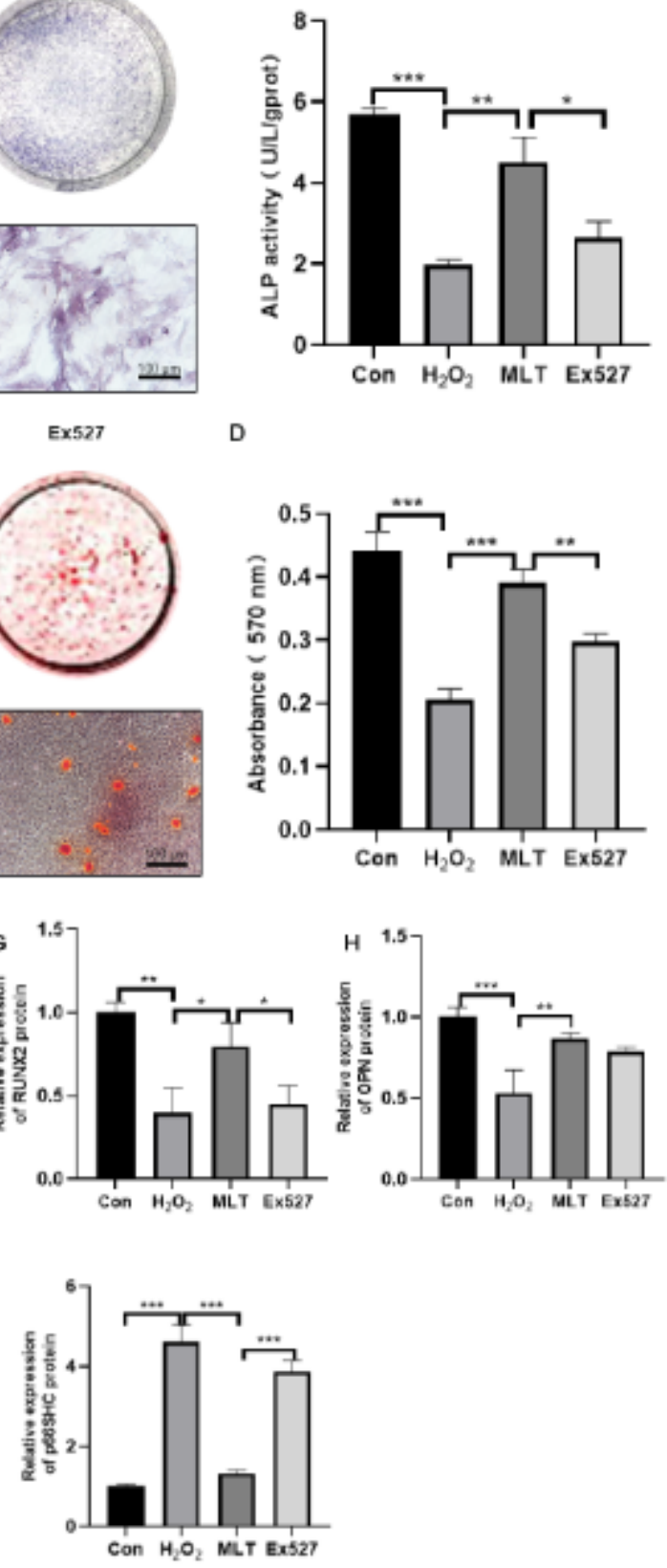

Figure 1 
Effect of melatonin on osteogenesis in MC3T3-E1 cells. (A) Alkaline phosphatase (ALP) staining on day 7 in the four groups. Scale bar $=100 \mu \mathrm{m}$. (B) Assessment of ALP activity in the four groups. (C) Alizarin Red $S$ (ARS) staining on day 21 in the four groups. Scale bar $=100 \mu \mathrm{m}$. (D) Calcium mineralization in the four groups quantified using a microplate reader. (E) Representative images showing the expression of the osteogenic proteins BMP2, RUNX2, OPN and SIRT1, p66SHC in MC3T3-E1 cells from the four groups. Values in (F-J) represent the fold change compared to the control. Con: cells were incubated in a-MEM alone; $\mathrm{H} 2 \mathrm{O} 2$ : cells were pretreated with $400 \mu \mathrm{M} \mathrm{H} 2 \mathrm{O} 2$ for $4 \mathrm{~h}$ and then incubated with a-MEM for $24 \mathrm{~h}$; MLT: cells were pretreated with $400 \mu \mathrm{M} \mathrm{H} 202$ for $4 \mathrm{~h}$ then incubated with $100 \mu \mathrm{M}$ melatonin for $24 \mathrm{~h}$; EX527: cells were pretreated with $400 \mu \mathrm{M} \mathrm{H} 2 \mathrm{O} 2$ for $4 \mathrm{~h}$, then incubated with $10 \mu \mathrm{M}$ EX527 for $2 \mathrm{~h}$ and 100 $\mu \mathrm{M}$ melatonin for $24 \mathrm{~h} .{ }^{*} \mathrm{P} \otimes 0.05,{ }^{*} \mathrm{P} \otimes 0.01, * * * P \otimes 0.001$.

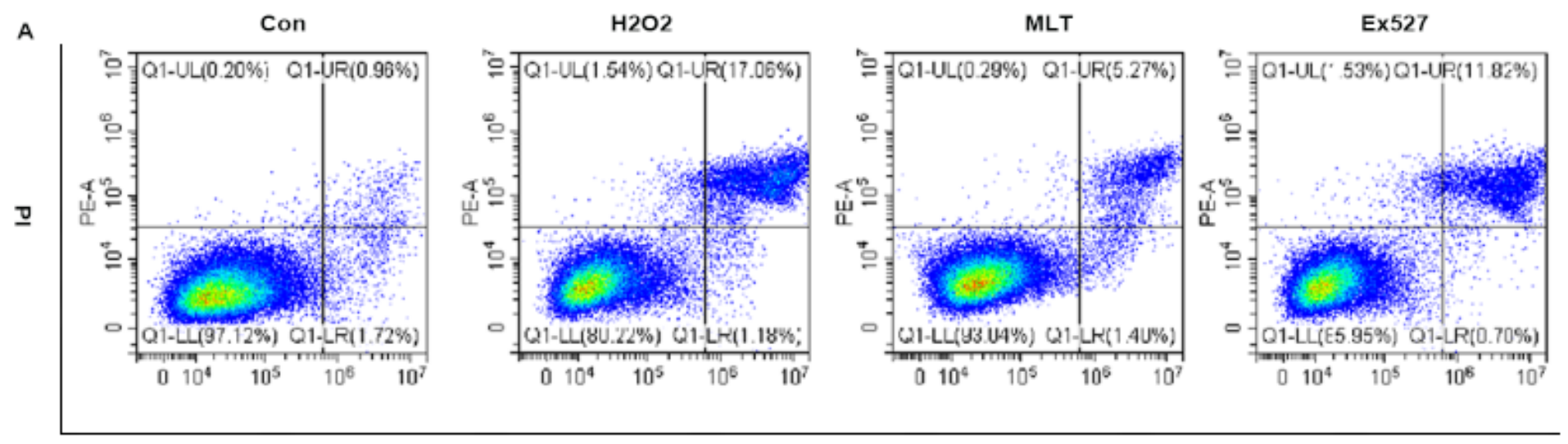

B

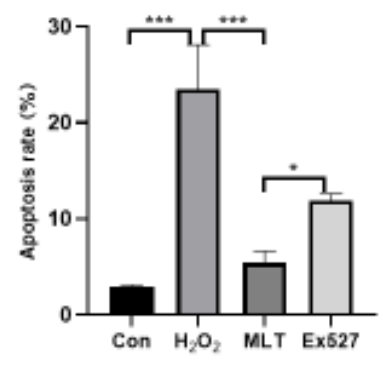

E

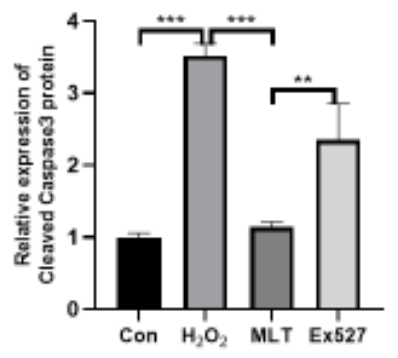

C

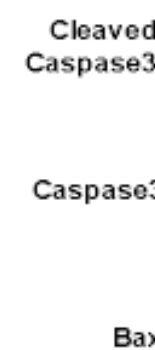

$\mathrm{Bax}$

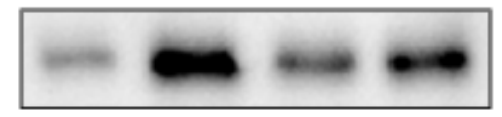

$\mathrm{BCl2}$

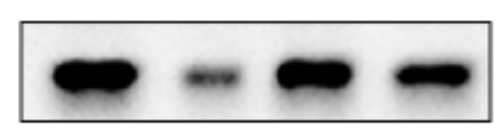

$\beta$-actin

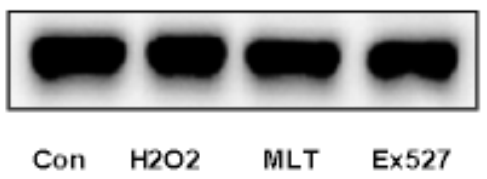

$17 \mathrm{kDa}$

$37 \mathrm{kDa}$

$21 \mathrm{kDa}$
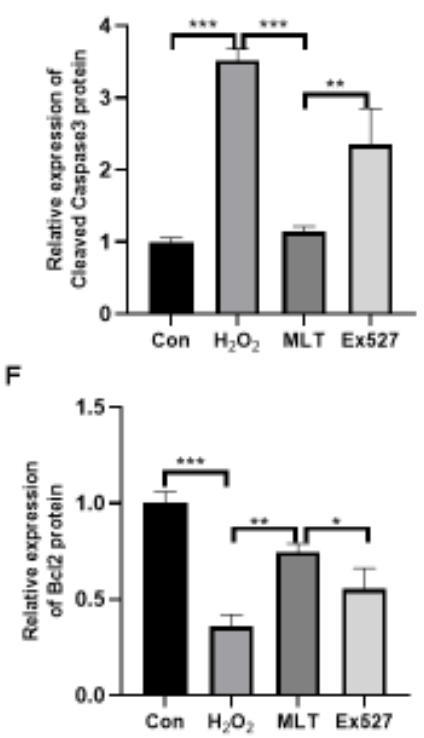

\section{Figure 2}

Protective effect of melatonin against the H2O2-induced apoptosis of MC3T3-E1 cells. (A) Flow cytometry analysis of Annexin V-FITC/PI double-stained MC3T3-E1 cells. (B) Comparison of apoptosis rates among the four groups. (C) Representative images showing the expression of the apoptotic proteins 
Bax, Bcl2, cleaved caspase3 and caspase3 in MC3T3-E1 cells from the four groups. Values in (D-F) represent the fold change compared to the control. *P囚0.05, **P囚0.01, ***P囚0.001.
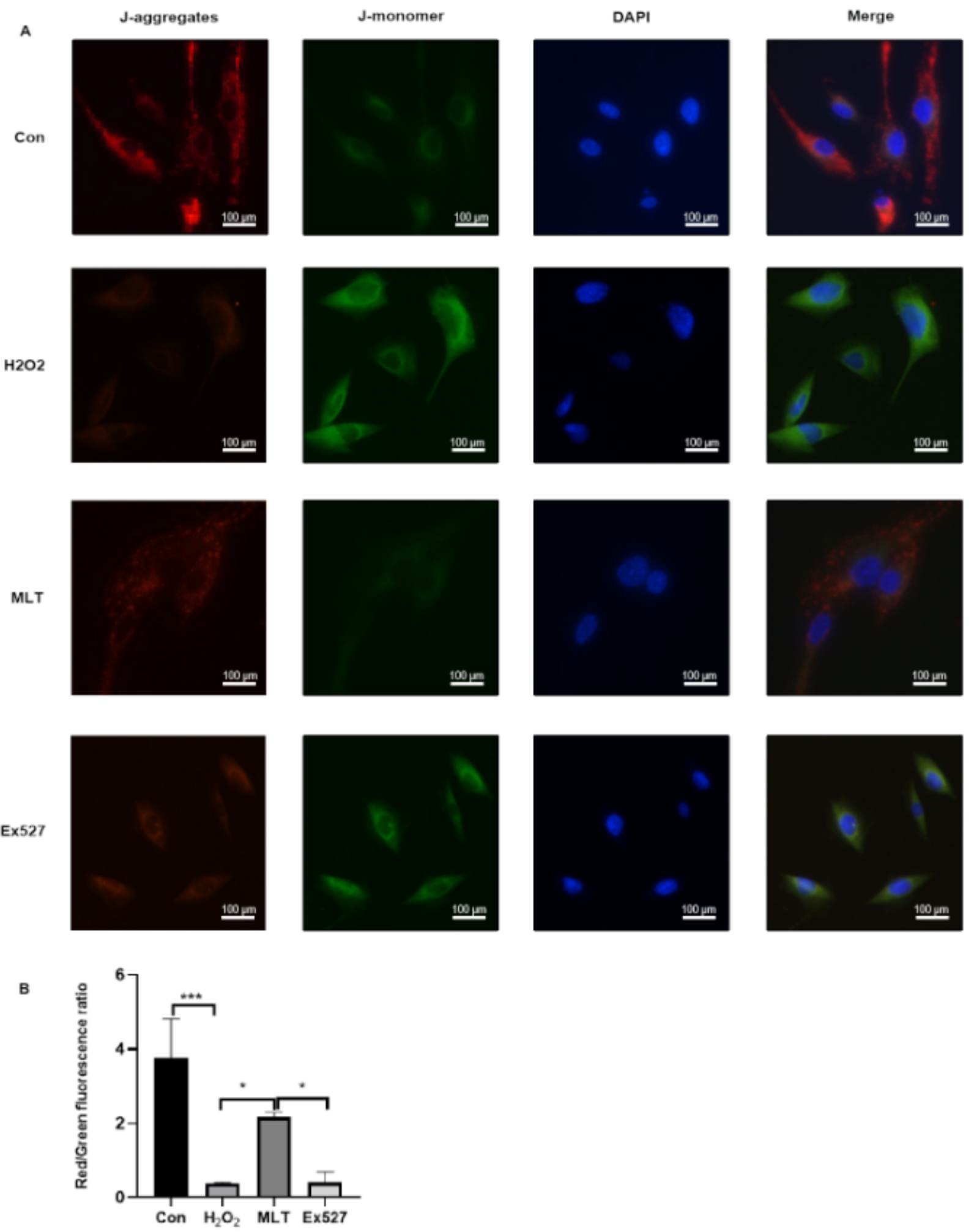

Figure 3

Melatonin inhibited the H2O2-induced mitochondrial membrane potential reduction in MC3T3-E1 cells. (A) Representative images of MC3T3-E1 cells stained for JC-1. Scale bar $=100 \mu \mathrm{m}$. (B) Quantitative analysis of JC-1 fluorescence in the four groups. ${ }^{*} \mathrm{P} \otimes 0.05,{ }^{* *} \mathrm{P} \otimes 0.01,{ }^{* * *} \mathrm{P} \otimes 0.001$. 
A

Con
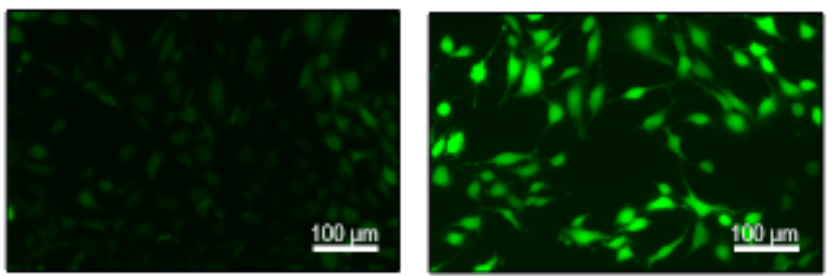

B

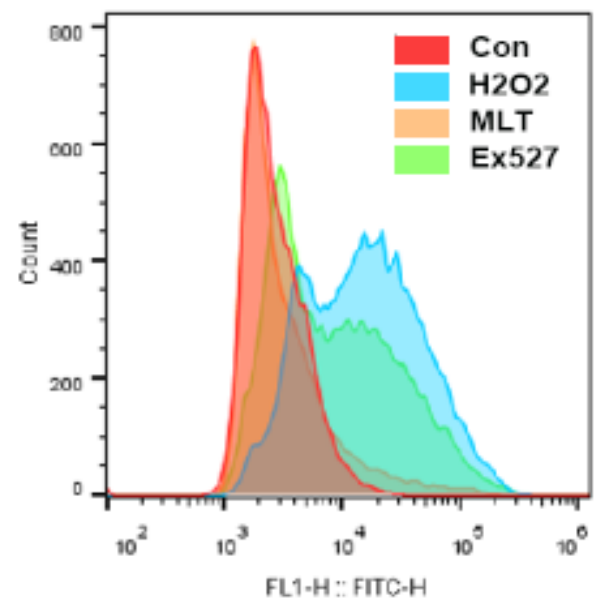

D

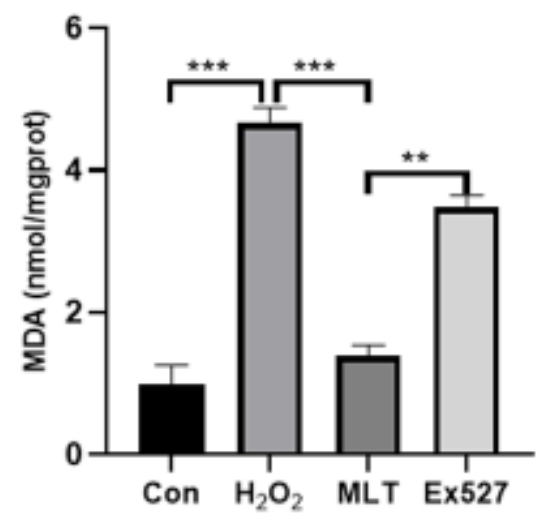

MLT

Ex527
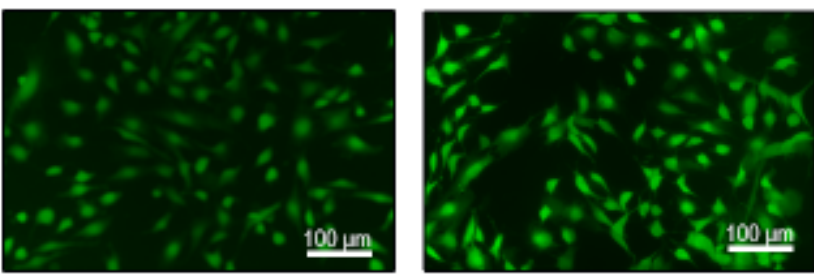

C

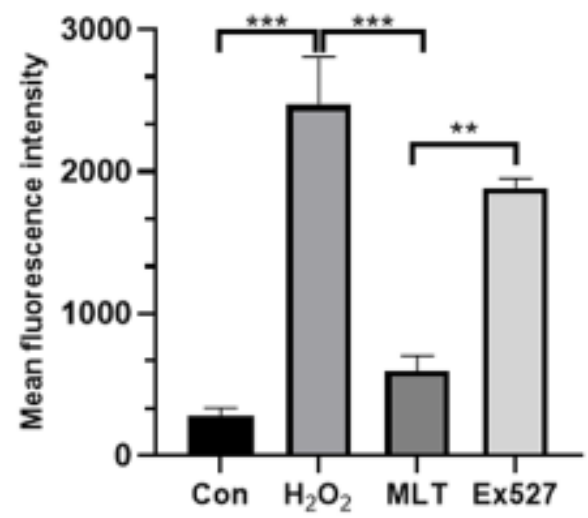

E



Figure 4

Melatonin enhances antioxidant capacity in H2O2-treated MC3T3-E1 cells. (A) Representative images of MC3T3-E1 cells labeled with the fluorescent probe DCFH-DA. Scale bar $=100 \mu \mathrm{m}$. (B) The mean fluorescence intensity of MC3T3-E1 cells labeled with the fluorescent probe DCFH-DA, determined using flow cytometry. (C) Quantitative analysis of the mean fluorescence intensity in the four groups. (D) Quantitative analysis of malondialdehyde (MDA) levels in the four groups. (E) Analysis of superoxide

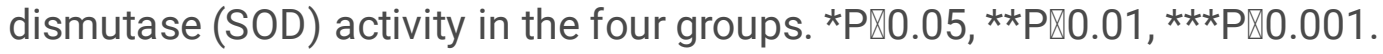


A
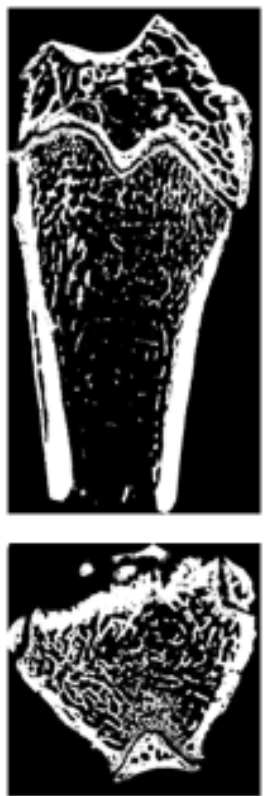

Sham
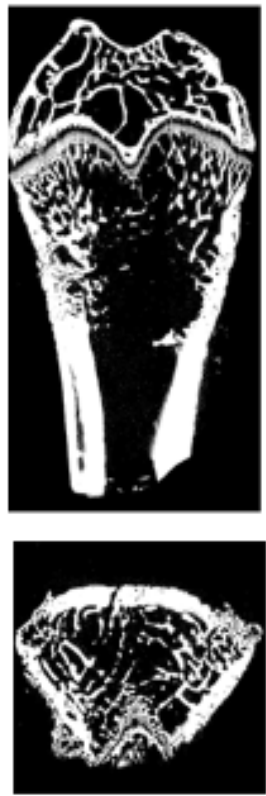

ovX
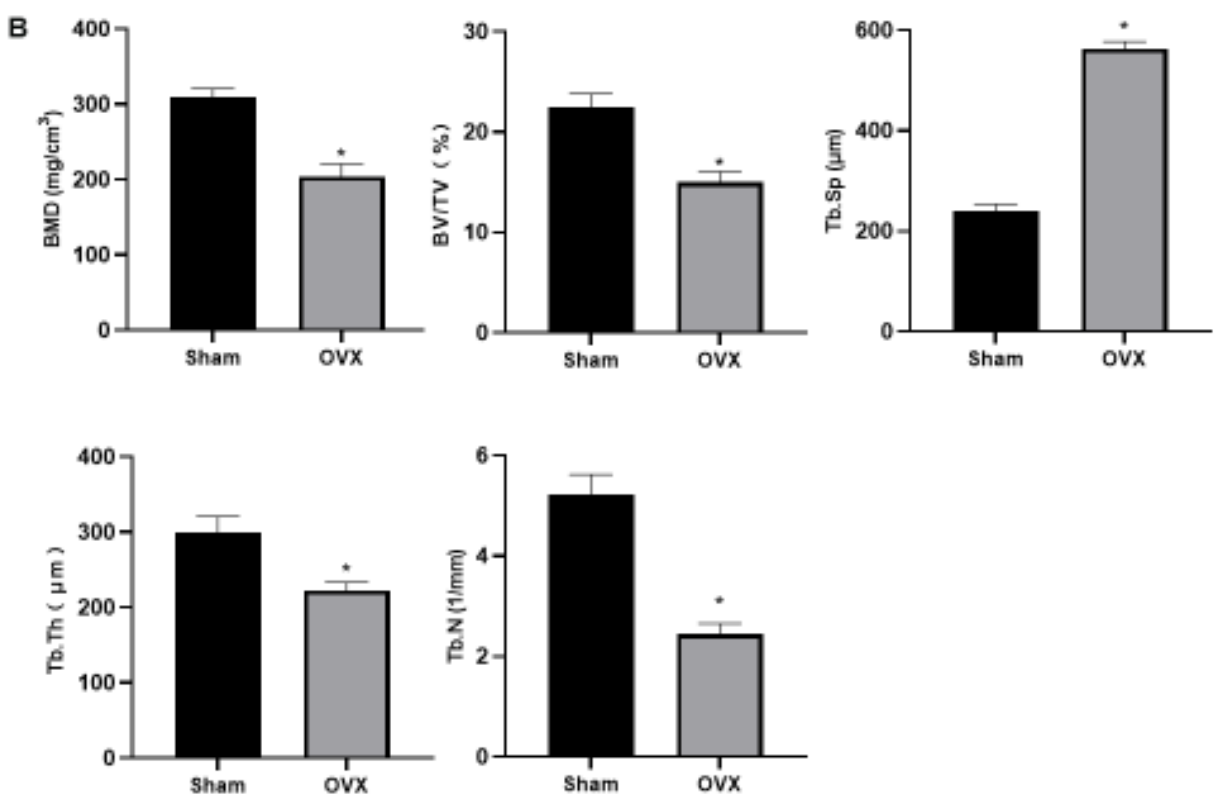

C

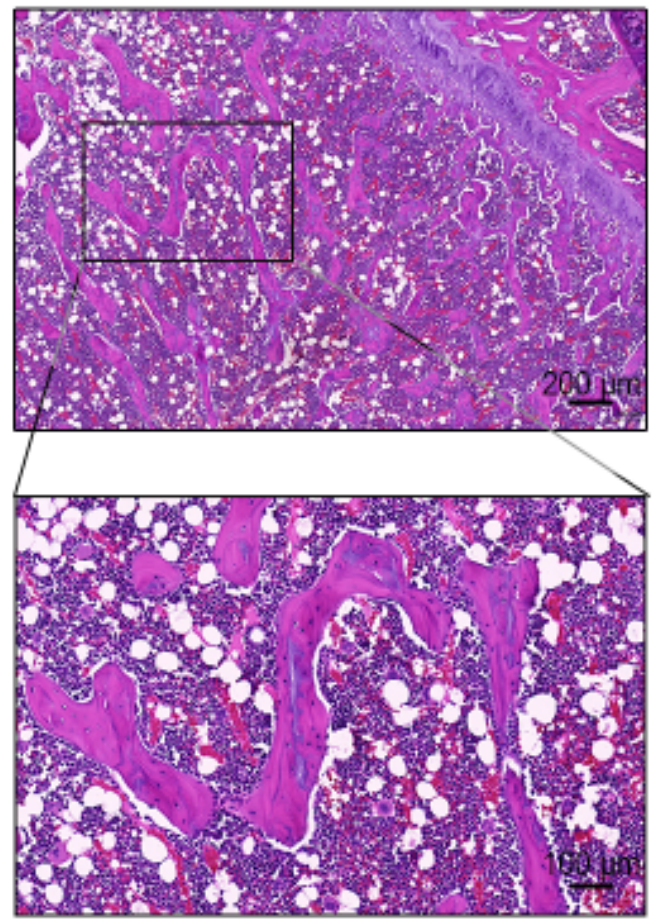

Sham

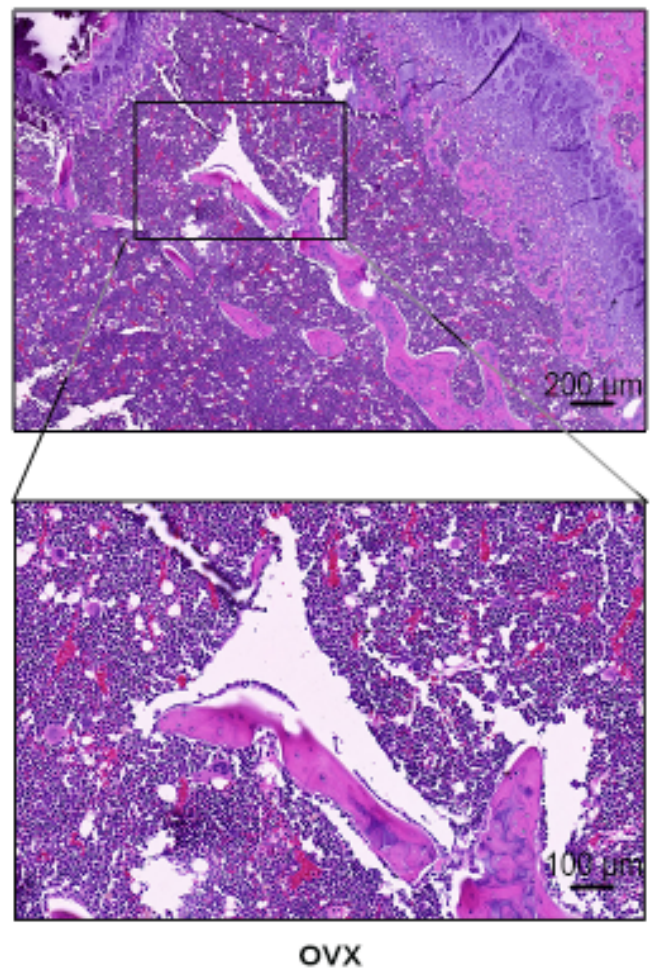

Figure 5

Establishment of the osteoporosis model. (A) Micro-computed tomography (micro-CT) analysis of femur metaphysis in the sham and OVX groups. (B) Quantitative analysis of femur metaphysis using micro-CT. (C) Representative hematoxylin-eosin staining of the distal femur in the sham group and OVX groups. BMD, bone mineral density; BV/TV, bone volume fraction; Tb.N, trabecular number; Tb.Th, trabecular thickness; Tb.Sp, trabecular separation. *P $\otimes 0.05$ compared to the sham group. 

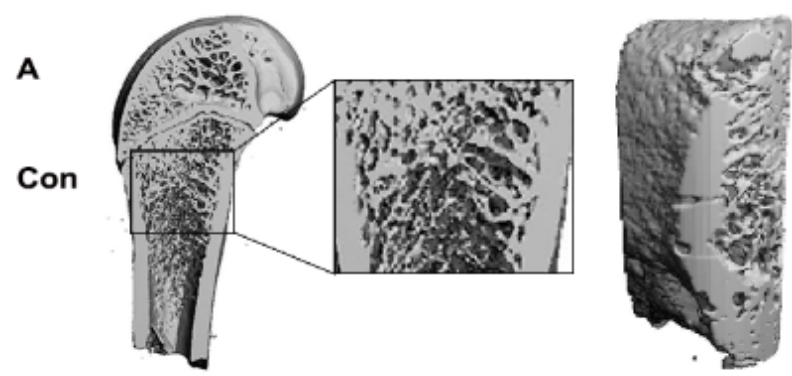

B

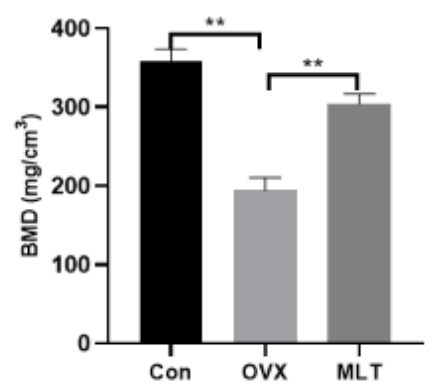

D

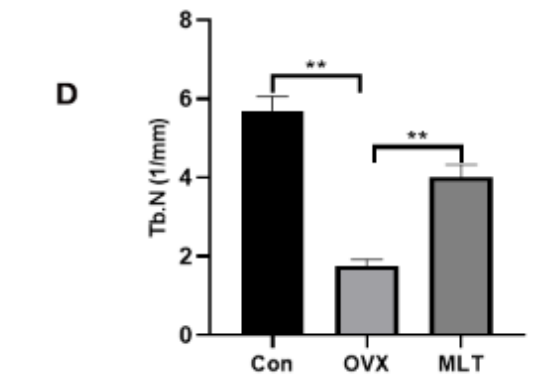

$\mathrm{F}$

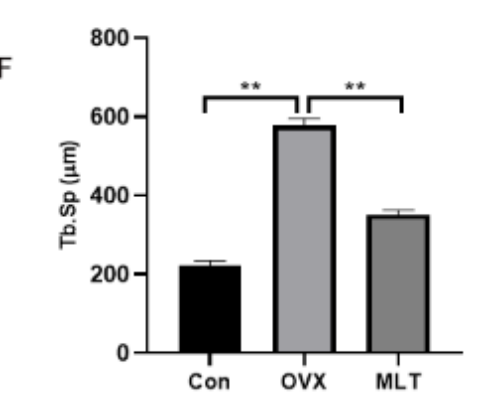

C
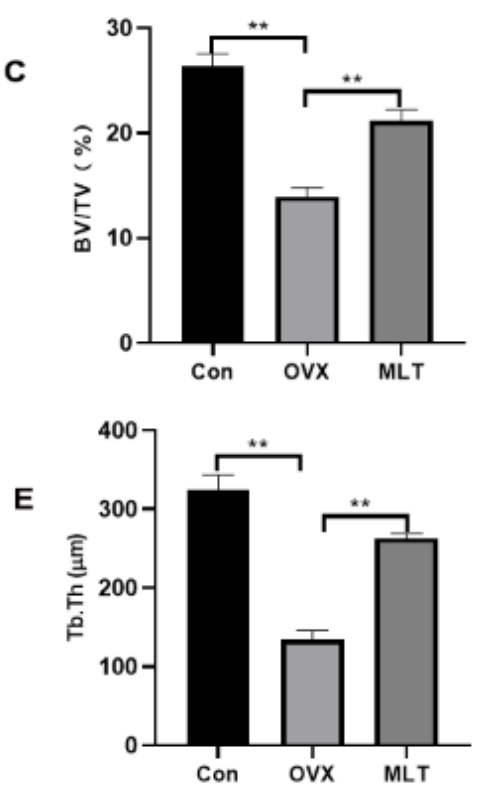

MLT
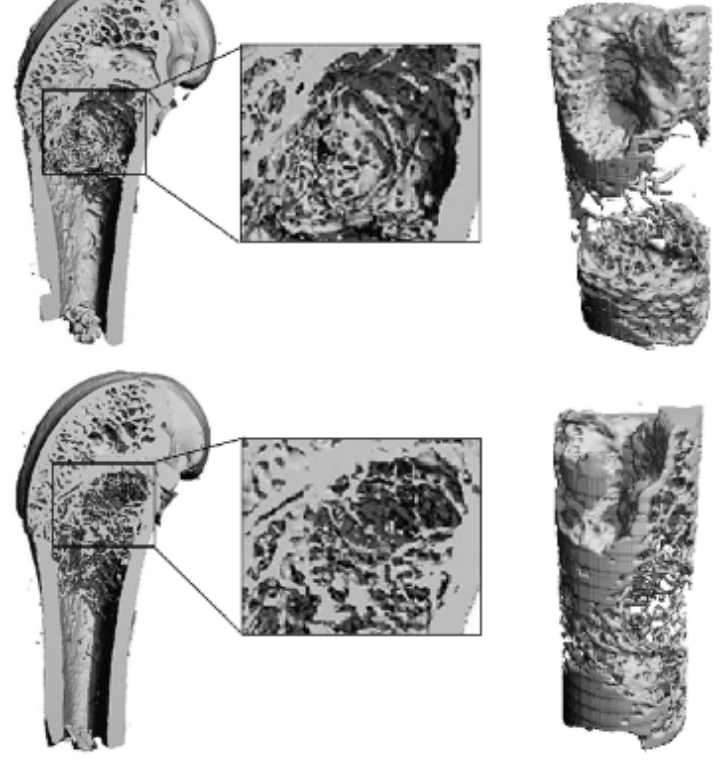

ovx

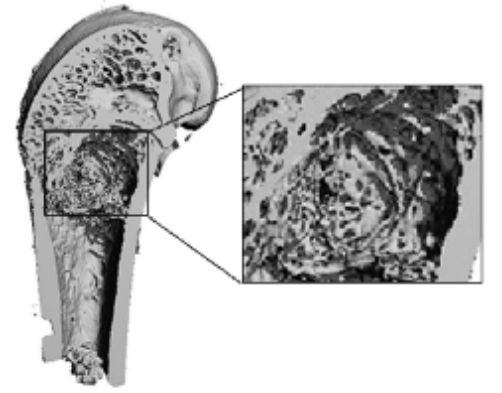

Figure 6

Melatonin promotes the healing of osteoporotic bone defects. (A) Three-dimensional reconstruction images of bone defects in the femur metaphysis. The left side of 3D image was a gross view of the femur, the center was a magnified view of the bone defect and the right cylinder shows a threedimensional composite of the bone defect. (B-F) Quantitative analysis of femur metaphysis using microCT. *Pख0.05, **P®0.01.

\section{Figure 7}

Melatonin promote new bone formation. (A and B) Hematoxylin-eosin and Masson's trichrome staining indicating the formation of new bone. Scale bar $=200 \mu \mathrm{m}$. (C and D) Rate of new bone formation observed using OCN and COL1 immunohistochemical staining. Scale bar $=50 \mu \mathrm{m}$. (E) Histogram showing the rate of new bone formation in the three groups. ( $F$ and $G$ ) Immunohistochemical quantitative analysis of the differences in OCN and COL1 expression among the three groups. TB: trabecular bone; BM: bone marrow; black circle: bone defect zone; asterisk: mature bone; pound sign: new bone and collagen fibers; black arrow: positive areas of immunohistochemistry staining. ${ }^{\star P} \rrbracket 0.05,{ }^{\star \star} \mathrm{P} \rrbracket 0.01,{ }^{\star \star \star} \mathrm{P} \rrbracket$ 0.001 . 